\title{
Singlet-Triplet Switching Induced by Electric Field in Triple Quantum Dots
}

\author{
J. ŁUCZAK* AND B.R. BUŁKA \\ Institute of Molecular Physics, Polish Academy of Sciences, M. Smoluchowskiego 17, 60-179 Poznań, Poland \\ We analyze influence of an external electric field on magnetic properties of a triple quantum dot system in \\ a triangular and linear geometry. The system contains four electrons and is described by an extended Hubbard \\ model which includes electron correlations and exchange processes. The electric field leads to splitting of energy \\ levels (the Stark effect) and to transition between the singlet and triplet states.
}

PACS: 71.10.--w, 33.57.+c, 73.23.-b, 73.63.Kv

\section{Introduction}

We present studies of an artificial molecule consisting of three coherently coupled quantum dots with four electrons which are placed in an external electric field. The electric field acting on the atoms or molecules causes a shift and splits of spectral lines. This effect is known in literature as the Stark effect. Our studies show that the electric field can also influence on magnetic properties of the system and one can see a transition between singlet and triplet states. The singlet-triplet $(\mathrm{S}-\mathrm{T})$ transition was investigated in quantum dot systems by many authors, as a function of magnetic field [1] or bias voltage [2]. Recently Baadji et al. [3] found that the $\mathrm{S}$ $\mathrm{T}$ transition can be induced by electric field in a real linear molecule. They studied, by means of the density functional theory (DFT), cobaltocene dimers, which contained two magnetic centers connected by an acetylene bridge. Adding different substituents to the molecule dielectric properties were changed and they observed the $\mathrm{S}-\mathrm{T}$ transition. In this paper we investigate the $\mathrm{S}-\mathrm{T}$ transition in a model of a three site molecule described by an extended Hubbard Hamiltonian, which takes into account electronic correlations and exchange processes. Analyzing the spectrum for the molecule with a triangular and a linear geometry we show that the singlet-triplet transition can occur for the triangular symmetry only. We cannot confirm the results for the linear molecule studied in [3] for any moderated values of parameters.

\section{Model}

Our model of triple quantum dots (TQD) (see Fig. 1) is described by an extended Hubbard model:

\footnotetext{
* corresponding author; e-mail: jakub.luczak@ifmpan.poznan.pl
}

$$
\begin{aligned}
\hat{H} & =\sum_{i, \sigma}\left[\epsilon_{i}+g_{E} \cos (\theta+(i-1) 2 \pi / 3)\right] n_{i \sigma} \\
& +\sum_{i \neq j, \sigma} t_{i j}\left(c_{i \sigma}^{\dagger} c_{j \sigma}+\text { H.c. }\right)+\sum_{i} U_{i} n_{i \downarrow} n_{i \uparrow} \\
& -\sum_{i \neq j} J_{i j} \boldsymbol{S}_{\boldsymbol{i}} \cdot \boldsymbol{S}_{\boldsymbol{j}},
\end{aligned}
$$

in which the first term shows influence of the electric field $\boldsymbol{E}$ on a local site energy $\epsilon_{i}$ in the $i$-th quantum dot. Here we assume that the polarization energy $\boldsymbol{E} \cdot \boldsymbol{P}=$ $e \sum_{i} \boldsymbol{E} \cdot \boldsymbol{r}_{i} n_{i}=\operatorname{Eer} \sum_{i} \cos (\theta+(i-1) 2 \pi / 3)$, where $e$ denotes the electron charge, $\boldsymbol{r}_{i}$ is a vector to the $i$-th quantum dot and $\theta$ is the angle between $\boldsymbol{r}_{1}$ and $\boldsymbol{E}$. The second term of the model (1) describes coherent electron hopping

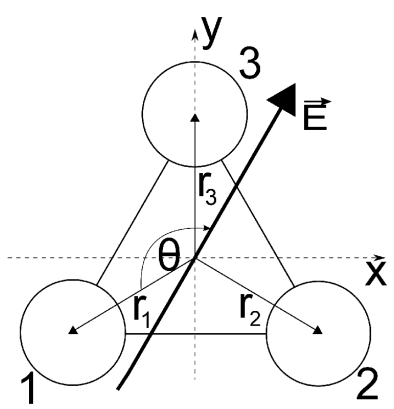

Fig. 1. Model of TQD placed in an external electric field $\boldsymbol{E}$.

between nearest quantum dots, the third term - local Coulomb interactions, whereas the last term - the direct exchange coupling between spins in the nearest-neighbor quantum dots. In general the exchange coupling $J_{i j}$ is positive and prefers a ferromagnetic spin configuration. For some special cases, for example, for different electron orbitals and their specific orientation, it can take a negative value. The Hubbard model takes into account elec- 
tronic correlations and contains (in an implicit way) also higher order exchange processes like a super- and double exchange coupling [4]. An explicit form of the superexchange coupling $J_{\text {super }}$ can be obtained by a canonical transformation $[5,6]$. It is however very laborious task, especially for the present case because one should make calculations to a 4th order perturbation [7]. We therefore, perform an exact numerical analysis of the spectrum and other properties of the system.

Moreover we assume that the system is isolated and contains four electrons. For a real molecule placed between electrodes, constant number of electrons can be hold in a wide range of parameters for fixed electrochemical potentials. For our case the singlet state with the total spin $S=0$ is expressed as

$$
\begin{aligned}
|S\rangle & =\frac{x_{12}^{S}}{\sqrt{2}} c_{3 \uparrow}^{\dagger} c_{3 \downarrow}^{\dagger}\left(c_{1 \uparrow}^{\dagger} c_{2 \downarrow}^{\dagger}-c_{1 \downarrow}^{\dagger} c_{2 \uparrow}^{\dagger}\right)|\mathrm{vac}\rangle \\
+ & \frac{x_{13}^{S}}{\sqrt{2}} c_{2 \uparrow}^{\dagger} c_{2 \downarrow}^{\dagger}\left(c_{1 \uparrow}^{\dagger} c_{3 \downarrow}^{\dagger}-c_{1 \downarrow}^{\dagger} c_{3 \uparrow}^{\dagger}\right)|\mathrm{vac}\rangle \\
+ & \frac{x_{23}^{S}}{\sqrt{2}} c_{1 \uparrow}^{\dagger} c_{1 \downarrow}^{\dagger}\left(c_{2 \uparrow}^{\dagger} c_{3 \downarrow}^{\dagger}-c_{2 \downarrow}^{\dagger} c_{3 \uparrow}^{\dagger}\right)|\mathrm{vac}\rangle \\
& +\sum_{i \neq j \neq k} x_{k}^{S} c_{i \uparrow}^{\dagger} c_{j \uparrow}^{\dagger} c_{i \downarrow}^{\dagger} c_{j \downarrow}^{\dagger}|\mathrm{vac}\rangle
\end{aligned}
$$

and the triplet state with $S=1$

$$
\begin{gathered}
|T\rangle=\frac{x_{12}^{T}}{\sqrt{2}} c_{3 \uparrow}^{\dagger} c_{3 \downarrow}^{\dagger}\left(c_{1 \uparrow}^{\dagger} c_{2 \downarrow}^{\dagger}+c_{1 \downarrow}^{\dagger} c_{2 \uparrow}^{\dagger}\right)|\mathrm{vac}\rangle \\
+\frac{x_{13}^{T}}{\sqrt{2}} c_{2 \uparrow}^{\dagger} c_{2 \downarrow}^{\dagger}\left(c_{1 \uparrow}^{\dagger} c_{3 \downarrow}^{\dagger}+c_{1 \downarrow}^{\dagger} c_{3 \uparrow}^{\dagger}\right)|\mathrm{vac}\rangle \\
+\frac{x_{23}^{T}}{\sqrt{2}} c_{1 \uparrow}^{\dagger} c_{1 \downarrow}^{\dagger}\left(c_{2 \uparrow}^{\dagger} c_{3 \downarrow}^{\dagger}+c_{2 \downarrow}^{\dagger} c_{3 \uparrow}^{\dagger}\right)|\mathrm{vac}\rangle .
\end{gathered}
$$

Here we present the triplet for $S_{z}=0$ only, because the case for $S_{z}= \pm 1$ is obvious. The coefficients $\left|x_{i j}^{S / T}\right|^{2}$ and $\left|x_{k}^{S}\right|^{2}$ are derived from the Schödinger equation.

\section{Results and discussion}

Let us first consider a fully symmetric molecule with the triangular geometry. Influence of the electric field on the energy of the two lowest states is shown in Fig. 2. Here we denote $g_{E}=e E r$. The top panels $(\mathrm{a}, \mathrm{b})$ are plotted for $t=1$. For $J_{d}=0$ the ground state is singlet in the whole range of $g_{E}$ and the singlet-triplet transition does not occur (Fig. 2a). Figure $2 \mathrm{~b}$ is plotted for a large value of the direct exchange $J_{d}=0.8$, which prefers the ferromagnetic spin configuration. As a result, the energy of the singlet state is shifted up, whereas the energy of triplet state goes down. At $g_{E}=0$ the ground state is singlet and the $\mathrm{S}-\mathrm{T}$ transition appears at $g_{E} \approx 3.4$. However with an increase of the electric field another transition occurs at $g_{E} \approx 6.6$ (not shown in the figure) and the ground state becomes again singlet.

The bottom panels in Fig. 2 present the position of the singlet and the triplet states for the hopping integral $t=-1$. Here at $g_{E}=0$ the ground state is triplet. Now we have a transition from the triplet to the singlet state, which occurs at $g_{E} \approx 2.6$ (Fig. $2 \mathrm{c}$ ). As one can expect the triplet-singlet transition is shifted to a higher value of $g_{E}$ when $J_{d}$ increases. For the parameters in Fig. 2d the transition appears at $g_{E} \approx 4.5$. The $\mathrm{S}-\mathrm{T}$ transition is induced by the electric field and it is cause by competition between the direct and superexchange processes.

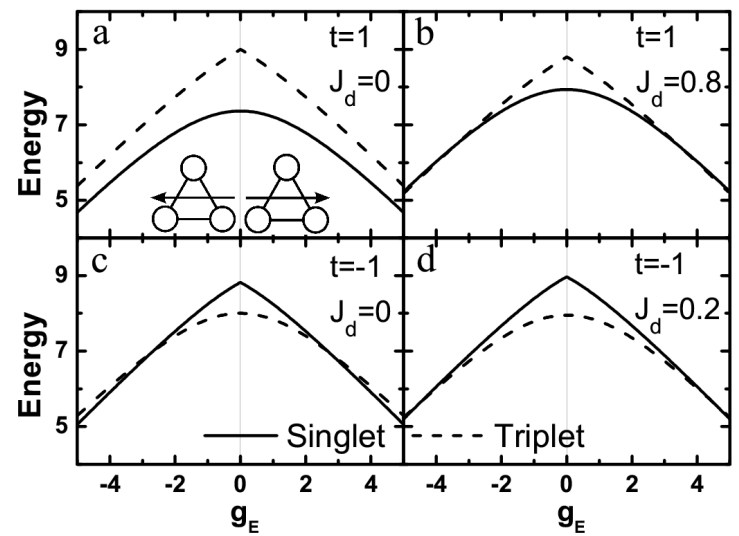

Fig. 2. Singlet and triplet lowest state as a function of the electric field for fully symmetric molecule: $\epsilon_{1}=\epsilon_{2}=$ $\epsilon_{3}=0, U_{1}=U_{2}=U_{3}=10, t_{12}=t_{13}=t_{23}=t, J_{12}=$ $J_{13}=J_{23}=J_{d}$. The positive value of $g_{E}$ corresponds to $\theta=7 \pi / 6$ and negative for opposite direction $\theta=\pi / 6$ (insets in plot a). The values of $t$ and $J_{d}$ are shown in the plots.

Comparing the top and the bottom panels in Fig. 2 one can see that the ground state of the triangular molecule depends on the sign of the hopping parameter $t$. For a negative value of $t$ the ground state is triplet, while for $t>0$ the ground state is singlet. It is related with the electron-hole asymmetry in the triangular system and different mobilities of the singlet and the triplet valence bonds (see also [1]).

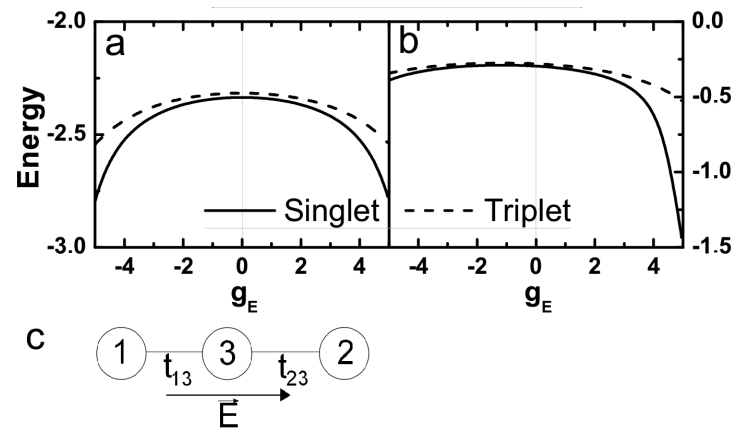

Fig. 3. Singlet and triplet lowest state as a function of electric field for a linear symmetric molecule (a) with $\epsilon_{1}=\epsilon_{2}=0$ and a linear asymmetric molecule (b) with $\epsilon_{1}=0, \epsilon_{2}=2$. The other parameters are taken as $t_{12}=0, t_{13}=t_{23}=1, U_{i}=10, J_{i j}=0, \epsilon_{3}=-6$. The same results are obtained for $t_{13}=t_{23}=-1$. 
Let us now consider the linear molecule $M_{1}-B-M_{2}$, composed of two magnetic centers $M_{1}$ and $M_{2}$ connected by a bridge $B$ (Fig. 3c). For the case studied by Baadji [3] the magnetic centers are represented by cobaltocene (or its derivative), which interact by the acetylene bridge. In our model we assume that the central dot 3 is diamagnetic with double electron occupation. Plots of the singlet and the triplet lowest state with respect to the electric field are presented in Fig. $3 a$ and b, for the symmetric and asymmetric case, respectively. Here the asymmetry of the molecule is modeled by a difference in local site energies at the quantum dot 1 and 2 . We have also investigated asymmetric molecules taking different hoping parameters $t_{13} \neq t_{23}$, different Coulomb interaction parameters $U_{1} \neq U_{2}$ and direct exchange couplings $J_{13} \neq J_{23}$. For any set of moderate parameters the ground state is singlet and the S-T transition does not occur. Moreover one can see in Fig. 3 that the difference between the singlet and the triplet state increases with $g_{E}$ which means that the antiferromagnetic interaction increases as well. We cannot confirm the results by Baadji et al. [3], where the transition from the singlet to the triplet state was induced by electric field.

\section{Summary}

Summarizing, we investigated influence of the electric field on magnetic properties of the triple quantum dot system with four electrons. We performed exact numerical calculations for the ground state within the extended Hubbard model taking into account electron correlations and exchange processes. Two geometries were analyzed: a triangular and linear molecule. We predict transitions from the singlet to triplet state and from the triplet to singlet state induced by electric field in the triangular molecule. However for the linear molecule the ground state is singlet for any moderate values of parameters and the singlet-triplet transition does not occur. One can expect that the predicted singlet-triplet switching effects will be seen in measurements of electronic transport due to the Pauli spin blockade [8]. Our model is general and can be used for quantum dots, real molecular systems as well as strong correlated electrons on lattices with a triangular symmetry. The effects predicted can be applied in new devices for spintronics and quantum computing.

\section{Acknowledgments}

This work was supported by Ministry of Science and Higher Education (Poland) from sources for science in the years 2009-2012 and by the EU project Marie Curie ITN NanoCTM.

\section{References}

[1] F. Delgado, Y.-P. Shim, M. Korkusinski, P. Hawrylak, Phys. Rev. B 76, 115332 (2007); F. Delgado, Y.-P. Shim, M. Korkusinski, L. Gaudreau, S.A. Studenikin, A. S. Sachrajda, P. Hawrylak, Phys. Rev. Lett. 101, 226810 (2008); Y.-P. Shim, F. Delgado, P. Hawrylak, Phys. Rev. B 80, 115305 (2009).

[2] H. Ribeiro, G. Burkard, Phys. Rev. Lett. 102, 216802 (2009).

[3] N. Baadji, M. Piacenza, T. Tugsuz, F.D. Sala, G. Maruccio, S. Sanvito, Nature Mater. 8, 813 (2009).

[4] K. Yosida, Theory of Magnetism, Springer, Berlin 1996.

[5] K. A. Chao, J. Spałek, A. M. Oleś, J. Phys. C 10, L271 (1977); K. A. Chao, J. Spalek, A. M. Oles, Phys. Rev. B 18, 3453 (1978).

[6] T. Kostyrko, B.R. Bułka, Phys. Rev. B 84, 035123 (2011).

[7] H. Eskes, J. H. Jefferson, Phys. Rev. B 48, 9788 (1993).

[8] See for example: J. Danon, Yu. V. Nazarov, Phys. Rev. B 80, 041301 (2009). 